\title{
Divisibility of qubit channels and dynamical maps
}

\author{
David Davalos ${ }^{1}$, Mario Ziman ${ }^{2,3}$, and Carlos Pineda $a^{1,4}$ \\ ${ }^{1}$ Instituto de Física, Universidad Nacional Autónoma de México, México, D.F., México \\ ${ }^{2}$ Institute of Physics, Slovak Academy of Sciences, Dúbravská cesta 9, Bratislava 84511, Slovakia \\ ${ }^{3}$ Faculty of Informatics, Masaryk University, Botanická 68a, 60200 Brno, Czech Republic \\ ${ }^{4}$ Faculty of Physics, University of Vienna, 1090 Vienna, Austria \\ January 22, 2020
}

The concept of divisibility of dynamical maps is used to introduce an analogous concept for quantum channels by analyzing the simulability of channels by means of dynamical maps. In particular, this is addressed for Lindblad divisible, completely positive divisible and positive divisible dynamical maps. The corresponding L-divisible, CP-divisible and Pdivisible subsets of channels are characterized (exploiting the results by Wolf et al. [25]) and visualized for the case of qubit channels. We discuss the general inclusions among divisibility sets and show several equivalences for qubit channels. To this end we study the conditions of L-divisibility for finite dimensional channels, especially the cases with negative eigenvalues, extending and completing the results of Ref. [26]. Furthermore we show that transitions between every two of the defined divisibility sets are allowed. We explore particular examples of dynamical maps to compare these concepts. Finally, we show that every divisible but not infinitesimal divisible qubit channel (in positive maps) is entanglement breaking, and open the question if something similar occurs for higher dimensions.

\section{Introduction}

The advent of quantum technologies opens questions aiming for deeper understanding of the fundamental physics beyond the idealized case of isolated quantum systems. Also the well established Born-Markov approximation used to describe open quantum systems (e.g. relaxation process such as spontaneous decay) is of limited use and a more general framework of open system dynamics is demanded. Recent efforts in this area have given rise to relatively novel research subjects - non-markovianity and divisibility.

Non-markovianity is a characteristic of continuous time evolutions of quantum systems (quantum dynamical maps), whereas divisibility refers to properties of system's transformations (discrete quantum

David Davalos: davidphysdavalos@gmail.com processes) over a fixed time interval (quantum channels). The non-markovianity aims to capture and describe the back-action of the system's environment on the system's future time evolution. Such phenomena is identified as emergence of memory effects [1, 18, 22]. On the other side, the divisibility questions the possibility of splitting a given quantum channel into a concatenation of other quantum channels. In this work we will investigate the relation between these two notions.

Our goal is to understand the possible forms of the dynamics standing behind the observed quantum channels, specially in regard to their divisibility properties which in turn determine their markovian or nonmarkovian nature. In particular, we provide characterization of the subsets of qubit channels depending on their divisibility properties and implementation by means of dynamical maps. An attempt to characterize the set of channels belonging to one-parameter semigroups induced by (time-independent) Lindblad master equations has been already done in Ref. [26]. However, it has drawbacks when dealing with channels with negative eigenvalues. Using the results of Ref. [5] and Ref. [3], we will extend the analysis of [26] also for channels with negative eigenvalues.

The paper is organized as follows: In section 2 we give the formal definition of quantum channels and of quantum dynamical maps, and some of their properties. We discuss the meaning of divisibility for each object and discuss the known inclusions and equivalences between divisibility types. In section 3 we discuss properties and representations of qubit channels and their divisibility. We introduce a useful theorem to decide L-divisibility, which is in turn valid for any finite dimension. In section 4 we discuss the possible transition that can be occur between divisibility types, and show two examples of dynamical maps and their transitions. Finally in Section 5 we summarize our results and discuss open questions. 


\section{Basic definitions and divisibility}

\subsection{Channels and divisibility classes}

We shall study transformations of a physical system associated with a complex Hilbert space $\mathcal{H}_{d}$ of dimension $d$. In particular, we consider linear maps on bounded operators, $\mathcal{B}\left(\mathcal{H}_{d}\right)$, that for the finitedimensional case coincides with the set of trace-class operators that accommodate the subset of density operators representing the quantum states of the system. We say a linear map $\mathcal{E}: \mathcal{B}\left(\mathcal{H}_{d}\right) \rightarrow \mathcal{B}\left(\mathcal{H}_{d}\right)$ is positive, if it maps positive operators into positive operators, i.e. $X \geq 0$ implies $\mathcal{E}[X] \geq 0$. Quantum channels are associated with elements of the convex set $\mathrm{C}$ of completely positive trace-preserving linear maps (CPTP) transforming density matrices into density matrices, i.e. $\mathcal{E}: \mathcal{B}(\mathcal{H}) \rightarrow \mathcal{B}(\mathcal{H})$ such that $\operatorname{tr}(\mathcal{E}[X])=\operatorname{tr}(X)$ for all $X \in \mathcal{B}(\mathcal{H})$, and all its extensions $\mathrm{id}_{n} \otimes \mathcal{E}$ are positive maps for all $n>1$, where $\mathrm{id}_{n}$ is the identity channel on a $n$-dimensional quantum system. In general a channel has the form $\mathcal{E}[X]=\sum_{i} K_{i} X K_{i}^{\dagger}$. The minimum number of operators $K_{i}$ required in the previous expression is called the Kraus rank of $\mathcal{E}$.

Let us introduce two subsets of channels. First, we say a channel is unital if it preserves the identity operator, i.e. $\mathcal{E}[\mathbb{1}]=\mathbb{1}$. Unital channels have a simple parametrization which will be useful for our purposes. Second, if $\mathcal{E}[X]=U X U^{\dagger}$ for some unitary operator $U$ (meaning $U U^{\dagger}=U^{\dagger} U=\mathbb{1}$ ), we say the channel is unitary.

A quantum channel $\mathcal{E}$ is called indivisible if it cannot be written as a concatenation of two non-unitary channels, namely, if $\mathcal{E}=\mathcal{E}_{1} \mathcal{E}_{2}$ implies that either $\mathcal{E}_{1}$, or $\mathcal{E}_{2}$, exclusively, is a unitary channel. If the channel is not indivisible, it is said to be divisible. We denote the set of divisible channels by $\mathrm{C}^{\text {div }}$ and that of indivisible channels by $\overline{\mathrm{C}^{\mathrm{div}}}$. Following this definition, unitary channels are divisible, because for them both (decomposing) channels $\mathcal{E}_{1,2}$ must be unitary. The concept of indivisible channels resembles the concept of prime numbers: unitary channels play the role of unity (which are not indivisible/prime), i.e. a composition of indivisible and a unitary channel results in an indivisible channel.

We now define the set of infinitely divisible channels $\left(\mathrm{C}^{\infty}\right)$ and the set of infinitesimal divisible channels (C Inf $)$. Infinitely divisible channels, in some sense opposite to indivisible channels, are defined as channels $\mathcal{E}$ for which there exist for all $n=1,2,3, \ldots$ a channel $\mathcal{A}_{n}$ such that $\mathcal{E}=\left(\mathcal{A}_{n}\right)^{n}$. Now, consider channels $\mathcal{E}$ that may be written as products of channels close to identity, i.e. such that for all $\epsilon>0$ there exists a finite set of channels $\varepsilon_{j}$ with $\|$ id $-\varepsilon_{j} \| \leq \epsilon$ and $\mathcal{E}=\prod_{j} \varepsilon_{j}$. Its closure determines the set of infinitesimal divisible channels $\mathrm{C}^{\mathrm{Inf}}$.

\subsection{Quantum dynamical maps and more divis- ibility classes}

The next sets of channels are going to be defined using three types of dynamical maps. A quantum dynamical map is identified with a continuous parametrized curve drawn inside the set of channels starting at the identity channel, i.e. a one-parametric function $t \mapsto \mathcal{E}_{t} \in \mathrm{C}$ for all $t$ belonging to an interval with minimum element 0 and satisfying the initial condition $\mathcal{E}_{0}=$ id. Let $\mathcal{E}_{t, s}=\mathcal{E}_{t}^{-1} \mathcal{E}_{s}$ be the linear map describing the state transformations within the time interval $[t, s]$, whenever $\mathcal{E}_{t}^{-1}$ exists.

- A given quantum dynamical map is called $C P$ divisible if for all $t<s$ the map $\mathcal{E}_{t, s}$ is a channel.

- A given quantum dynamical map is called $P$ divisible [22] if $\mathcal{E}_{t, s}$ is a positive trace-preserving linear map for all $t<s$.

- A given quantum dynamical map is called $L$ divisible if it is induced by a time-independent Lindblad master equation $[7,14,16]$, i.e. $\mathcal{E}_{t}=e^{t L}$ with

$$
L(\rho)=i[\rho, H]+\sum_{\alpha, \beta} G_{\alpha \beta}\left(F_{\alpha} \rho F_{\beta}^{\dagger}-\frac{1}{2}\left\{F_{\beta}^{\dagger} F_{\alpha}, \rho\right\}\right),
$$

where $H=H^{\dagger} \in \mathcal{B}\left(\mathcal{H}_{d}\right)$ is known as Hamiltonian, $\left\{F_{\alpha}\right\}$ are hermitian and form an orthonormal basis of the operator space $\mathcal{B}\left(\mathcal{H}_{d}\right)$, and $G_{\alpha \beta}$ constitutes a hermitian positive semi-definite matrix.

If we allow the Lindblad generator $L$ to depend on time, we recover the set of CP-divisible quantum dynamical maps as the resulting dynamical maps $\mathcal{E}_{t}=\hat{\mathrm{T}} e^{\int_{0}^{t} L(\tau) d \tau}(\hat{\mathrm{T}}$ denotes the time-ordering operator) are compositions of infinitesimal completelypositive maps $[1,7,14,16]$. Notice that there is a hierarchy for quantum dynamical maps: L-divisible quantum dynamical maps are $C P$-divisible which in turn are P-divisible.

Using the introduced families of quantum dynamical maps we can now classify quantum channels according to whether they can be implemented by the aforementioned kinds of quantum dynamical maps. We define subsets $\mathrm{C}^{\mathrm{L}}, \mathrm{C}^{\mathrm{CP}}$, and $\mathrm{C}^{\mathrm{P}}$ of L-divisible, $C P$-divisible and $P$-divisible channels, respectively. In particular, we say $\mathcal{E} \in \mathrm{C}^{\mathrm{L}}$ if it belongs to the closure of a L-divisible quantum dynamical map. Let us stress that the requirement of the existence of Lindblad generator $L$ such that $\mathcal{E}=e^{L}$ is not sufficient and closure is necessary. For example, the evolution governed by $L(\rho)=i[\rho, H]+\gamma[H,[H, \varrho]]$ results [27] in the diagonalization of states in the energy eigenbasis of $H$. Such transformation $\mathcal{E}_{\text {diag }}$ is not invertible, thus (by definition) $L=\log \mathcal{E}_{\text {diag }}$ does not exist (contains infinities). Analogously, we say $\mathcal{E} \in \mathrm{C}^{\mathrm{CP}}\left(\mathcal{E} \in \mathrm{C}^{\mathrm{P}}\right)$ 
if there exists a CP-divisible (P-divisible) dynamical map $\mathcal{E}_{t}$ such that $\mathcal{E}=\mathcal{E}_{t}$ (with arbitrary precision) for some value of $t$ (including $t=\infty$ ).

We now recall how to verify whether a channel is L-divisible since we will build upon the method for some of our results. Verifying whether $\mathcal{E} \in \mathrm{C}^{\mathrm{L}}[26]$ requires evaluation of the channel's logarithms, however, the matrix logarithm is defined only for invertible matrices and it is not unique. In fact, we need to check if at least one of its branches has the Lindblad form. It was shown in [5] that $\mathcal{E}$ is L-divisible if and only if there exists $L$ such that: $\exp L=\mathcal{E}$, is hermitian preserving, trace-preserving and conditionally completely positive (ccp). Thus, we are looking for logarithm satisfying $L\left(X^{\dagger}\right)=L(X)^{\dagger}$ (hermiticity preserving), $L^{*}(\mathbb{1})=0$ (trace-preserving), and

$$
(\mathbb{1}-\omega)(\mathrm{id} \otimes L)[\omega](\mathbb{1}-\omega) \geq 0
$$

(ccp condition), where $\omega=\frac{1}{d} \sum_{j, k=1}^{d}|j \otimes j\rangle\langle k \otimes k|$ is the projector onto a maximally entangled state. To the best of our knowledge, there is no general method to verify if a channel is $\mathrm{P}$ or $\mathrm{CP}$ divisible, with some exceptions [25].

\subsection{Relation between channel divisibility classes}

Due to the inclusion set relations between the three kinds of dynamical maps discussed in the previous sections, we can see that $\mathrm{C}^{\mathrm{L}} \subset \mathrm{C}^{\mathrm{CP}} \subset \mathrm{C}^{\mathrm{P}}$. Similarly, from the earlier definitions, one can easily argue that $\mathrm{C}^{\infty} \subset \mathrm{C}^{\mathrm{Inf}} \subset \mathrm{C}^{\text {div }}$. By the definition of Ldivisibility it is trivial to see that in general $C^{L} \subset C^{\infty}$. Indeed Denisov has shown in [4] that infinitely divisible channels can be written as $\mathcal{E}=\mathcal{E}_{0} e^{L}$, with $L$ a Lindblad generator, and $\mathcal{E}_{0}$ idempotent operator such that $\mathcal{E}_{0} L \mathcal{E}_{0}=\mathcal{E}_{0} L$. Further, it was shown in Ref. [25] that $\mathcal{E} \in \mathrm{C}^{\mathrm{inf}}$ implies $\operatorname{det} \mathcal{E} \geq 0$ and also that $\mathcal{E}$ can be approximated by $\prod_{j} e^{L_{j}}$, i.e. $\mathrm{C}^{\mathrm{CP}}=\mathrm{C}^{\mathrm{inf}}$. In other words, the positivity of determinant is necessary for the channel to be (in the closure of) channels attainable by CP-divisible dynamical maps. In summary, we have the following relations between sets (see also Fig. 1):

$$
\begin{array}{llll}
\mathrm{C}^{\infty} & \subset \mathrm{C}_{\|}^{\mathrm{Inf}} \subset \mathrm{C}^{\text {div }} \\
\mathrm{C}^{\mathrm{L}} & \subset \mathrm{C}^{\mathrm{CP}} \subset \mathrm{C}^{\mathrm{P}} .
\end{array}
$$

The relation between $C^{P}$ and $C^{\text {div }}$ is unknown, although it is clear that $\mathrm{C}^{\mathrm{div}} \subset \mathrm{C}^{\mathrm{P}}$ is not possible since channels in $\mathrm{C}^{\mathrm{P}}$ are not necessarily divisible in $\mathrm{CP}$ maps. The intersection of $\mathrm{C}^{\mathrm{P}}$ and $\mathrm{C}^{\mathrm{div}}$ is not empty since $\mathrm{C}^{\mathrm{CP}} \subseteq \mathrm{C}^{\text {div }}$ and $\mathrm{C}^{\mathrm{CP}} \subseteq \mathrm{C}^{\mathrm{P}}$. Later on we will investigate if $\mathrm{C}^{\mathrm{P}} \subseteq \mathrm{C}^{\text {div }}$ or not.

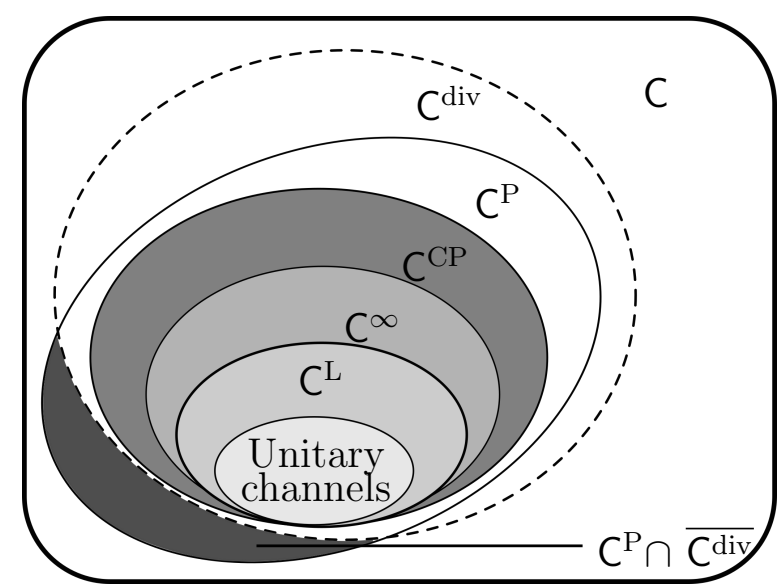

Figure 1: Scheme illustrating the different sets of quantum channels for a given dimension, discussed in sec. 2. In particular, the inclusion relations presented in Eq. (2) are depicted.

\section{Qubit channels}

\subsection{Representations}

Using the Pauli basis $\frac{1}{\sqrt{2}}\left\{\mathbf{1}, \sigma_{x}, \sigma_{y}, \sigma_{z}\right\}$, and the standard Hilbert-Schmidt inner product, the real representation for qubit channels is given by [10, 20]:

$$
\hat{\mathcal{E}}=\left(\begin{array}{cc}
1 & \overrightarrow{0}^{T} \\
\vec{t} & \Delta
\end{array}\right)
$$

This describes the action of the channel in the Bloch sphere picture in which the points $\vec{r}$ are identified with density operators $\varrho_{\vec{r}}=\frac{1}{2}(I+\vec{r} \cdot \vec{\sigma})$. We will write $\mathcal{E}=(\Delta, \vec{t})$ meaning that $\mathcal{E}\left(\rho_{\vec{r}}\right)=\rho_{\Delta \vec{r}+\vec{t}}$.

In order to study qubit channels with simpler expressions, we will consider a decomposition in unitaries such that

$$
\mathcal{E}=\mathcal{U}_{1} \mathcal{D U}_{2}
$$

This can be performed by decomposing $\Delta$ in rotation matrices, i.e. $\Delta=R_{1} D R_{2}$, where $D=$ $\operatorname{diag}\left(\lambda_{1}, \lambda_{2}, \lambda_{3}\right)$ is diagonal and the rotations $R_{1,2} \in$ $\mathrm{SO}(3)$ (of the Bloch sphere) correspond to the unitary channels $\mathcal{U}_{1,2}$. This decomposition should not be confused with the singular value decomposition. The latter allows decompositions that include, say, total reflections. Such operations do not correspond to unitaries over a qubit, in fact they are not CPTP. Therefore the channel $\mathcal{D}$, in the Pauli basis, is given by

$$
\hat{\mathcal{D}}=\left(\begin{array}{cc}
1 & \overrightarrow{0}^{T} \\
\vec{\tau} & D
\end{array}\right),
$$

where $\Delta=R_{1} D R_{2}$ and $\vec{\tau}=R_{1}^{T} \vec{t}$. The latter describes the shift of the center of the Bloch sphere under the action of $\mathcal{D}$. The parameters $\vec{\lambda}$ determine the length of semi-axes of the Bloch ellipsoid, being the deformation of Bloch sphere under the action of $\mathcal{E}$. From 
now we will call the form $\mathcal{D}$, special orthogonal normal form.

We shall develop a geometric intuition in the space determined by the possible values of these three parameters. For an arbitrary channel, complete positivity implies that the possible set of lambdas lives inside the tetrahedron with corners $(1,1,1),(1,-1,-1)$, $(-1,1,-1)$ and $(-1,-1,1)$, see Fig. 2. For unital channels, all points in the tetrahedron are allowed, but for non-unital channels more restrictive conditions arise. In Fig. 8 we present a visualization of the permitted values of $\vec{\lambda}$ for a particular nontrivial value of $\vec{\tau}$, and in [2] the steps to study the general case from an algebraic point of view are presented. For the unital case, the corner $\vec{\lambda}=(1,1,1)$ corresponds to the identity channel, $\vec{\lambda}=(1,-1,-1)$ to $\sigma_{x} \vec{\lambda}=(-1,1,-1)$ to $\sigma_{y}$ and $\vec{\lambda}=(-1,-1,1)$ to $\sigma_{z}$ (Kraus rank 1 operations). Points in the edges correspond to Kraus rank 2 operations, points in the faces to Kraus rank 3 operations and in the interior of the tetrahedron to Kraus rank 4 operations.

In addition to this decomposition, following the definition of divisibility, concatenation with unitaries of a given quantum channel do not change the divisibility character of the latter. Thus, orthogonal normal forms are useful to study divisibility since, following also the properties of $\mathrm{C}^{\mathrm{P}}$ and $\mathrm{C}^{\mathrm{CP}}$ introduced in sec. 3.1, immediately one has:

Theorem 1 (Divisibility of special orthogonal normal forms). Let $\mathcal{E}$ a qubit quantum channel and $\mathcal{D}$ its special orthogonal normal form, $\mathcal{E}$ belongs to $\mathrm{C}^{X}$ if and only if $\mathcal{D}$ does, where $X=$ $\{$ "Div", " $P$ ", "CP" $\}$.

There is another another parametrization for qubit channels called Lorentz normal decomposition [23, 24] which is specially useful to characterize infinitesimal divisibility $\mathrm{C}^{\mathrm{Inf}}$, and geometric aspects of entanglement [15]. This decomposition is derived from the theorem 3 of Ref. [24], which essentially states that for a qubit state $\rho=\frac{1}{4} \sum_{i, j=0}^{3} R_{i j} \sigma_{i} \otimes \sigma_{j}$ the matrix $R$ can be decomposed as $R=L_{1} \Sigma L_{2}^{\mathrm{T}}$. Here $L_{1,2}$ are proper orthochronous Lorentz transformations and $\Sigma$ is either $\Sigma=\operatorname{diag}\left(s_{0}, s_{1}, s_{2}, s_{3}\right)$ with $s_{0} \geq s_{1} \geq s_{2} \geq\left|s_{3}\right|$, or

$$
\Sigma=\left(\begin{array}{cccc}
a & 0 & 0 & b \\
0 & d & 0 & 0 \\
0 & 0 & -d & 0 \\
c & 0 & 0 & -b+c+a
\end{array}\right)
$$

In theorem 8 of Ref. [23] the authors make a similar claim, exploiting the Choi-Jamiołkowski isomorphism. They forced $b=0$ in order to have normal forms proportional to trace-preserving operations in the case of Kraus rank deficient ones, see Eq. (6). The latter is equivalent to saying that the decomposition of Choi-Jamiołkowski states leads to states that are proportional to Choi-Jamiołkowski states. We didn't find a good argument to justify such an assumption

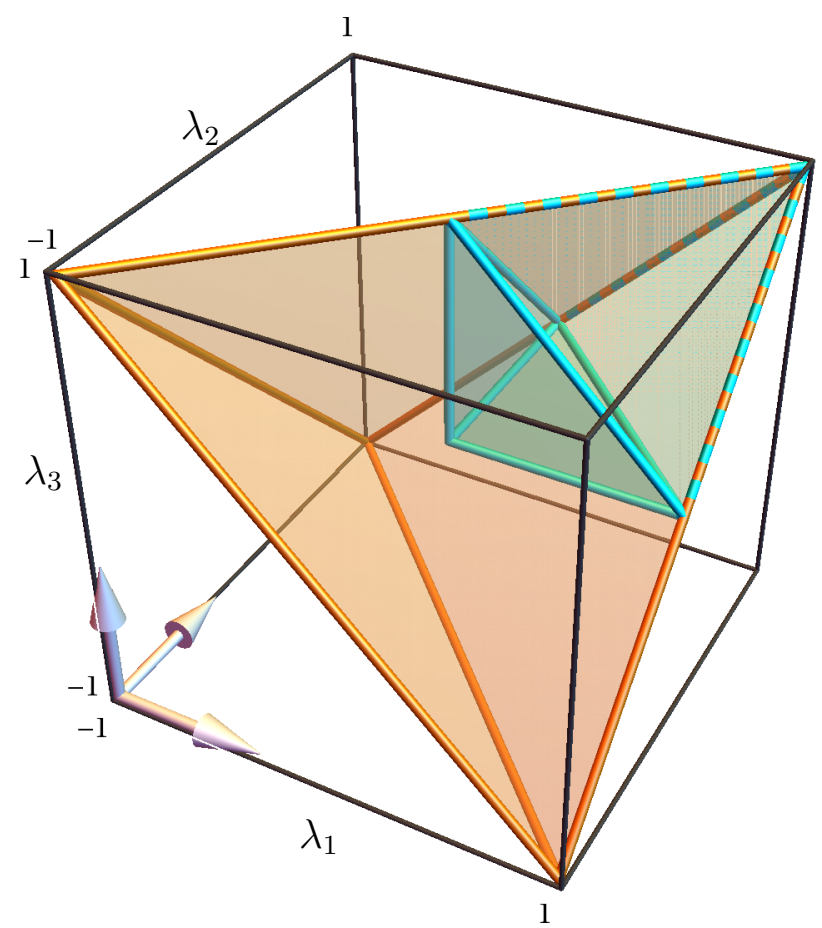

Figure 2: Tetrahedron of Pauli channels. The corners correspond to unitary Pauli operations $\left(\mathbb{1}, \sigma_{x, y, z}\right)$ while the rest can be written as convex combinations of them. The bipyramid in blue corresponds to channels with $\lambda_{i}>0 \forall i$, i.e. channels of the positive octant belonging to $C^{P}$. The whole set $C^{P}$ includes three other bipyramids corresponding to the other vertexes of tetrahedron, i.e. $C^{P}$ enjoys the symmetries of the tetrahedron, see Eq. (8). The faces of the bipyramids matching the corners of the tetrahedron are subsets of the faces of the tetrahedron, i.e. contain Kraus rank three channels. Such channels are then $C^{P}$ but also $\overline{C^{\text {div }}}$, showing that the intersection shown in Fig. 1 is not empty. 
and found a counterexample (see appendix A). Thus, we propose a restricted version of their theorem:

Theorem 2 (Restricted Lorentz normal form for qubit quantum channels). For any full Kraus rank qubit channel $\mathcal{E}$ there exists rank-one completely positive maps $\mathcal{T}_{1}, \mathcal{T}_{2}$ such that $\mathcal{T}=\mathcal{T}_{1} \mathcal{E} \mathcal{T}_{2}$ is proportional to

$$
\left(\begin{array}{cc}
1 & \overrightarrow{0}^{T} \\
\overrightarrow{0} & \Lambda
\end{array}\right)
$$

where $\Lambda=\operatorname{diag}\left(s_{1}, s_{2}, s_{3}\right)$ with $1 \geq s_{1} \geq s_{2} \geq\left|s_{3}\right|$.

The channel $\mathcal{T}$ is called the Lorentz normal form of the channel $\mathcal{E}$. For unital qubit channels $D$ coincides with $\Lambda$, thus in such case the form of (7) holds for any Kraus rank.

\subsection{Divisibility}

In this subsection we will recall the criteria to decide if a qubit channel belongs to $\mathrm{C}^{\text {div }}, \mathrm{C}^{\mathrm{P}}$ and $\mathrm{C}^{\mathrm{CP}}$ following [25]. We shall start with some general statements, and then focus on different types of channels (unital, diagonal non-unital and general ones). We will also discuss in detail the characterization of $\mathrm{C}^{\mathrm{L}}$, which entails a higher complexity.

It was shown ([25], Theorem 11) that full Kraus rank channels are divisible $\left(\mathrm{C}^{\mathrm{div}}\right)$. This simply means that all points in the interior of the set of channels correspond to divisible channels. Moreover, according to Theorem 23 of the same reference, qubit channels are indivisible if and only if they have Kraus rank three and diagonal Lorentz normal form. Notice that since we dispute the theorem upon which such statement is based, the classification might be inaccurate, see the appendix. It follows from the definition that for qubit channels $\mathcal{E}$ is divisible if and only if $\mathcal{D}$ is divisible. To test if $\mathcal{D}$ is divisible, we check that all eigenvalues of its Choi matrix are different from zero.

A non-negative determinant of $\mathcal{E}$ is a necessary condition for a general channel to belong to $\mathrm{C}^{\mathrm{P}}$ ([25], Proposition 15). For qubits, this is also sufficient ([25], Theorem 25), and given that $\operatorname{det} \mathcal{D}=\operatorname{det} \mathcal{E}$, the condition for qubit channels simply reads

$$
\operatorname{det} \mathcal{E}=\lambda_{1} \lambda_{2} \lambda_{3} \geq 0
$$

However, to our knowledge, a simple condition for arbitrary dimension is yet unknown.

With respect to testing for CP-divisibility we restrict the discussion to qubit channels. To characterize CP-divisible channels it is useful to consider the Lorentz normal form for channels. A full Kraus rank qubit channel $\mathcal{E}$ belongs to $\mathrm{C}^{\mathrm{CP}}$ if and only if it has diagonal Lorentz normal form with

$$
s_{\min }^{2} \geq s_{1} s_{2} s_{3}>0
$$

where $s_{\text {min }}$ is the smallest of $s_{1}, s_{2}$ and $s_{3}$, see theorem 2 and [26]. For Kraus deficient channels the pertinent theorems are based on Kraus deficient Lorentz normal forms that according to our appendix should be reviewed.

Deciding L-divisibility, as mentioned above, is equivalent to proving the existence of a hermiticity preserving generator which additionally fulfills the ccp condition.

To prove the former we recall that every hermiticity preserving operator has a real matrix representation when choosing a hermitian basis. Since quantum channels preserve hermiticity, the problem is reduced on finding a real $\operatorname{logarithm} \log \hat{\mathcal{E}}$ given a real matrix $\hat{\mathcal{E}}$. This problem was already solved by Culver [3] who characterized completely the existence of real logarithms of real matrices. For diagonalizable matrices the results can be summarized as follows:

Theorem 3 (Existence of hermiticity preserving generator). A non-singular matrix with real entries $\hat{\mathcal{E}}$ has a real generator (i.e. a $\log \hat{\mathcal{E}}$ has real entries) if and only if the spectrum fulfills the following conditions:

(i) negative eigenvalues are even-fold degenerate;

(ii) complex eigenvalues come in complex conjugate pairs.

Let us examine this theorem for the particular case of qubits. In this case this theorem means that real $\operatorname{logarithm}(\mathrm{s})$ of $\hat{\mathcal{E}}$ exist if and only if $\mathcal{E}$ has either only positive eigenvalues, one positive and two complex, or one positive and two equal non-positive eigenvalues, apart from the trivial eigenvalue equal to one. Notice that quantum channels with complex eigenvalues will fulfill the last condition immediately since they preserve hermiticity.

We now continue discussing the multiplicity of the solutions of $\log \hat{\mathcal{E}}$, as finding an appropriate parametrization is essential to test for the ccp condition, see Eq. (1). If $\hat{\mathcal{E}}$ has positive degenerate, negative, or complex eigenvalues, its real logarithms are not unique, and are spanned by real logarithm branches [3]. In case of having negative eigenvalues, it turns out that real logarithms always have a continuous parametrization, in addition to real branches due to the freedom of the Jordan normal form transformation matrices. Given a real representation of $\mathcal{E}$, i.e. $\hat{\mathcal{E}}$, the Jordan form is given by $\hat{\mathcal{E}}=w J w^{-1}=\tilde{w} J \tilde{w}^{-1}$, where $w=\tilde{w} K$ with $K$ belonging to a continuum of matrices that commutes with $J$ [3]. In the case of diagonalizable matrices, if there are no degeneracies, $K$ commutes with $\log (J)$.

Finally let us note that if a channel belongs to $\mathrm{C}^{\mathrm{L}}$, unitary conjugations can bring it to $C^{\operatorname{Inf}} \backslash C^{L}$ and vice versa.

\subsection{Unital channels}

We shall start our study of unital qubit channels, by considering Pauli channels, defined as convex combi- 
nations of the unitaries $\sigma_{i}$ :

$$
\mathcal{E}_{\text {Pauli }}[\rho]=\sum_{i=0}^{3} p_{i} \sigma_{i} \rho \sigma_{i}
$$

where $\sigma_{0}=\mathbb{1}$ and $p_{i} \geq 0$ with $\sum_{i} p_{i}=1$. The special orthogonal normal form of a Pauli channel [see Eqs. (4) and (5)] has $\mathcal{U}_{1}=\mathcal{U}_{2}=\mathrm{id}$ and $\vec{\tau}=\overrightarrow{0}$. Thus, Pauli channels are fully characterized only by $\vec{\lambda}$. Notice that every unital qubit channel can be written as

$$
\mathcal{E}_{\text {unital }}=\mathcal{U}_{1} \mathcal{E}_{\text {Pauli }} \mathcal{U}_{2}
$$

This implies that arbitrary unital qubit channels can be expressed as convex combinations of (at most) four unitary channels.

Following theorem 1 it is straightforward to note that by characterizing $\mathrm{C}^{\text {div }}, \mathrm{C}^{\mathrm{P}}$ and $\mathrm{C}^{\mathrm{CP}}$ of Pauli channels, the same conclusions hold for general unital qubit channels having the same $\vec{\lambda}$. Additionally we can have a one-to-one geometrical view of the divisibility sets for Pauli channels given they have a one-to-one correspondence to the tetrahedron shown in Fig. 2, defined by the inequalities

$$
\begin{aligned}
& 1+\lambda_{i}-\lambda_{j}-\lambda_{k} \geq 0 \\
& 1+\lambda_{1}+\lambda_{2}+\lambda_{3} \geq 0
\end{aligned}
$$

with $i, j$ and $k$ all different [28].

\subsubsection{P-divisibility}

Let us discuss the divisibility properties of Pauli channels. Divisibility in CPTP ( $\mathrm{C}^{\text {div }}$ ) is guaranteed for full Kraus rank channels, i.e. for the interior channels of the tetrahedron. For Pauli channels this is equivalent to taking only the inequality of equations (13). The characterization of $\mathrm{C}^{\mathrm{P}}$ can be done directly using Eq. (8), as it depends only on $\vec{\lambda}$. This set is the intersection of the tetrahedron with the octants where the product of all $\lambda \mathrm{s}$ is positive. In fact, it consists of four triangular bipyramids starting in each vertex of the tetrahedron and meeting in its center, see Fig. 2. Let us study the intersection of this set with the set of unital entanglement breaking (EB) channels [28], forming an octahedron (being the intersection of the tetrahedron with its space inversion, see Fig. 3). It is defined by the inequalities

$$
\begin{aligned}
& \lambda_{1}+\lambda_{2}+\lambda_{3} \leq 1 \\
& \lambda_{i}-\lambda_{j}-\lambda_{k} \leq 1,
\end{aligned}
$$

with $i, j$ and $k$ all different [28], together with Eq. (13). It follows that unital qubit channels that are not achieved by P-divisible dynamical maps are necessarily entanglement breaking (see Fig. 3 and Fig. 7). In fact this holds for general qubit channels, see section 3.4 .

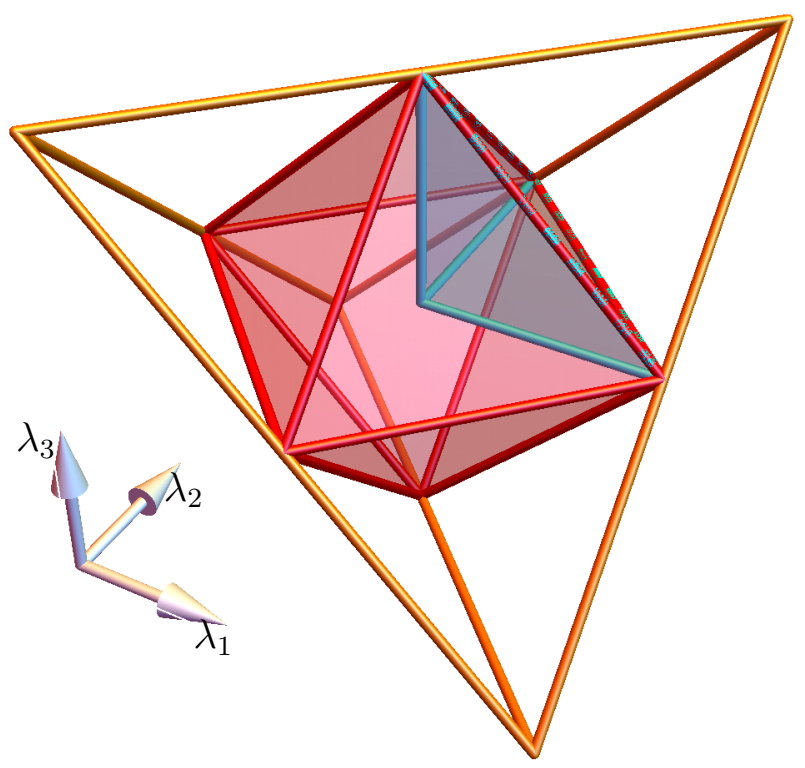

Figure 3: Tetrahedron of Pauli channels with the octahedron of entanglement breaking channels shown in red, see Eq. (14). The blue pyramid inside the octahedron is the intersection of the bipyramid shown in Fig. 2, with the octahedron. The complement of the intersections of the four bipyramids forms the set of divisible but not infinitesimal divisible channels in PTP. Thus, a central feature of the figure is that the set $C^{\text {div }} \backslash C^{P}$ is always entanglement breaking, but the converse is not true.

\subsubsection{CP-divisibility}

The subset of CP-divisible Pauli channels, following Eq. (9) and theorem 2, is determined by the inequalities

$$
0<\lambda_{1} \lambda_{2} \lambda_{3} \leq \lambda_{\min }^{2}
$$

They determine a body that is symmetric with respect to permutation of Pauli unitary channels (i.e. in $\lambda_{j}$ ), hence, the set of $\mathrm{C}^{\mathrm{CP}}$ of Pauli channels possesses the symmetries of the tetrahedron. The set $C^{C P} \backslash C^{L}$ is plotted in Fig. 5. Notice that this set coincides with the set of unistochastic qubit channels, see Ref. [17].

\subsubsection{L-divisibility}

Let us now derive the conditions for L-divisibility of Pauli channels with positive eigenvalues $\lambda_{1}, \lambda_{2}, \lambda_{3}$ $\left(\lambda_{0}=1\right)$. The logarithm of $\mathcal{D}$, induced by the principal logarithm of its eigenvalues, is thus

$$
L=K \operatorname{diag}\left(0, \log \lambda_{1}, \log \lambda_{2}, \log \lambda_{3}\right) K^{-1},
$$

which is real (hermiticity preserving). In case of nodegeneration the dependency on $K$ vanishes and $L$ is unique. In such case the ccp conditions $\log \lambda_{j}-$ $\log \lambda_{k}-\log \lambda_{l} \geq 0$ imply

$$
\lambda_{j} \lambda_{k} \leq \lambda_{l}
$$

for all combinations of mutually different $j, k, l$. This set (channels belonging to $\mathrm{C}^{\mathrm{L}}$ with positive eigenvalues) forms a three dimensional manifold, see Fig. 6. 
In case of degeneration, let us label the eigenvalues $\eta, \lambda$ and $\lambda$. In this case, the real solution for $L$ is not unique and is parametrized by real branches in the degenerate subspace and by the continuous parameters of $K$ [3]. Let us study the principal branch with $K=\mathbb{1}$. Eq. (17) is then reduced to

$$
\lambda^{2} \leq \eta \leq 1
$$

Therefore, if these inequalities are fulfilled, the generator has Lindblad form. If not, then a priori other branches can fulfill the ccp condition and consequently have a Lindblad form. Thus, Eq. (18) provides a sufficient condition for the channel to be in $\mathrm{C}^{\mathrm{L}}$. We will see it is also necessary.

Indeed, the complete positivity condition requires $\eta, \lambda \leq 1$, thus, it remains to verify only the condition $\lambda^{2} \leq \eta$. It holds for the case $\lambda \leq \eta$. If $\eta \leq \lambda$, then this condition coincides with the CP-divisibility condition from Eq. (15). Since $\mathrm{C}^{\mathrm{L}}$ implies $\mathrm{C}^{\mathrm{CP}}$ the proof is completed. In conclusion, the condition in Eq. (17) is a necessary and sufficient condition for a given Pauli channel with positive eigenvalues to belong to $\mathrm{C}^{\mathrm{L}}$.

Let us stress that the obtained subset of L-divisible channels does not possess the tetrahedron symmetries. In fact, composing $\mathcal{D}$ with a $\sigma_{z}$ rotation $\mathcal{U}_{z}=\operatorname{diag}(1,-1,-1,1)$ results in the Pauli channel $\mathcal{D}^{\prime}=\operatorname{diag}\left(1,-\lambda_{1},-\lambda_{2}, \lambda_{3}\right)$. Clearly, if $\lambda_{j}$ are positive ( $\mathcal{D}$ is L-divisible), then $\mathcal{D}^{\prime}$ has non-positive eigenvalues. Moreover, if all $\lambda_{j}$ are different, then $\mathcal{D}^{\prime}$ does not have any real logarithm, therefore, it cannot be L-divisible. In conclusion, the set of L-divisible unital qubit channels is not symmetric with respect to tetrahedron symmetries.

In what follows we will investigate the case of non-positive eigenvalues. Theorem 3 implies that that eigenvalues have the form (modulo permutations) $\eta,-\lambda,-\lambda$, where $\eta, \lambda \geq 0$. The corresponding Pauli channels are $\mathcal{D}_{x}=\operatorname{diag}(1, \eta,-\lambda,-\lambda)$, $\mathcal{D}_{y}=\operatorname{diag}(1,-\lambda, \eta,-\lambda), \mathcal{D}_{z}=\operatorname{diag}(1,-\lambda,-\lambda, \eta)$, thus forming three two-dimensional regions inside the tetrahedron. Take, for instance, $\mathcal{D}_{z}$ that specifies a plane (inside the tetrahedron) containing $I, \sigma_{z}$ and completely depolarizing channel $\mathcal{N}=\operatorname{diag}(1,0,0,0)$. The real logarithms for this case are given by

$L=K\left(\begin{array}{cccc}0 & 0 & 0 & 0 \\ 0 & \log (\lambda) & (2 k+1) \pi & 0 \\ 0 & -(2 k+1) \pi & \log (\lambda) & 0 \\ 0 & 0 & 0 & \log (\eta)\end{array}\right) K^{-1}$

where $k \in \mathbb{Z}$ and $K$, as mentioned above, belongs to a continuum of matrices that commute with $\mathcal{D}_{z}$. Note that $L$ is always non-diagonal. For this case (similarly for $\mathcal{D}_{x}$ and $\mathcal{D}_{y}$ ) the ccp condition reduces again to conditions specified in Eq. (18). Using the same arguments one arrives to a more general conclusion: Eq. (17) provides necessary and sufficient conditions for $L-$ divisibility of a given Pauli channel, and if it is the case, the principal branch with $K=\mathbb{1}$ has Lindblad form. The set of L-divisible Pauli channels is illustrated in Fig. 6.

In order to decide L-divisibility of general unital channels it remains to analyze the case of complex eigenvalues. The logarithms are parametrized as follows

$$
L_{k, K}=w K \log (J)_{k} K^{-1} w^{-1},
$$

where $J$ is the real Jordan form of the (qubit) channel [3]:

$$
J=\operatorname{diag}(1, c) \oplus\left(\begin{array}{cc}
a & -b \\
b & a
\end{array}\right)
$$

with $a \pm i b$ being the complex eigenvalues and $c>0$. Let us note that $K \log (J)_{k} K^{-1}$ is reduced to equations (16) and (19) in the case of real eigenvalues. In general, the generator is (up to diagonalization):

$$
\begin{aligned}
& K \log (J)_{k} K^{-1}=K \operatorname{diag}(0, \log (c)) \oplus \\
& \left(\begin{array}{cc}
\log (|z|) & \arg (z)+2 \pi k \\
-\arg (z)-2 \pi k & \log (|z|)
\end{array}\right) K^{-1} .
\end{aligned}
$$

with $z=a+i b$. The non-diagonal block of the logarithm has the same structure as the real Jordan form of the channel, so $K$ also commutes with $\log (J)_{k}$, leading to a countable parametric space of hermitian preserving generators. In fact, generators of diagonalizable channels have continuous parametrizations if and only if they have degenerate eigenvalues; the nondiagonalizable case can be found elsewhere [3]. Since we are dealing with a diagonalization, the ccp condition can be very complicated and depends in general on $k$, see Eq. (1). But for the complex case we can simplify the condition for qubit channels which have exactly the form presented in Eq. (21), say $\hat{\mathcal{E}}_{\text {complex }}$, i.e. $w=\mathbb{1}$. In such case the ccp condition is reduced to

$$
a^{2}+b^{2} \leq c \leq 1
$$

Note that it does not depend on $k$ and the second inequality is always fulfilled for CPTP channels.

We can present the conditions for L-divisibility for the case of complex eigenvalues. The orthogonal normal form of $\hat{\mathcal{E}}_{\text {complex }}$ is $\hat{\mathcal{D}}=\operatorname{diag}(1, \eta, \lambda, \lambda)$ with $\eta=c$ and $\lambda=\operatorname{sign}(a b) \sqrt{a^{2}+b^{2}}$. The ccp condition for degenerate eigenvalues, see Eq. (18), is reduced to Eq. (22) for this case. Therefore, the L-divisible channels with form $\hat{\mathcal{E}}_{\text {complex }}$ are also Ldivisible, up to unitaries. This also applies for channels arising from changing positions of the $1 \times 1$ block containing $c$ and the $2 \times 2$ block containing $a$ and $b$ in $\hat{\mathcal{E}}_{\text {complex }}$, with orthogonal normal forms $\operatorname{diag}(1, \lambda, \eta, \lambda)$ and $\operatorname{diag}(1, \lambda, \lambda, \eta)$. The set containing them is shown in Fig. 4.

\subsubsection{Divisibility relations}

Consider a Pauli channel with $0<\lambda_{\min }=\lambda_{1} \leq$ $\lambda_{2} \leq \lambda_{3}<1$, thus, the condition $\lambda_{1} \lambda_{2} \leq \lambda_{3}$ trivially holds. Since $\lambda_{1} \lambda_{2} \leq \lambda_{1} \lambda_{3} \leq \lambda_{2} \lambda_{3} \leq \lambda_{2}$, it 


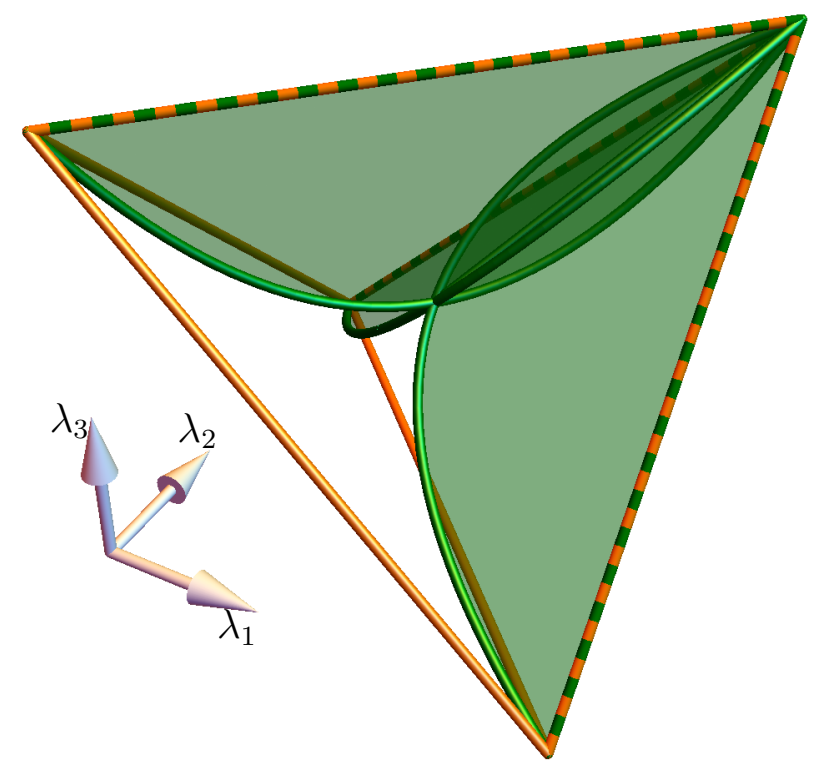

Figure 4: Tetrahedron of Pauli channels, with qubit unital L-divisible channels of the form $\hat{\mathcal{E}}_{\text {complex }}$ (see main text). Note that the set does not have the symmetries of the tetrahedron.

follows that $\lambda_{1} \lambda_{3} \leq \lambda_{2}$, thus, two (out of three) Ldivisibility conditions hold always for Pauli channels with positive eigenvalues. Moreover, one may observe that CP-divisibility condition Eq. (15) reduces to one of L-divisibility conditions $\lambda_{2} \lambda_{3} \leq \lambda_{1}$. In conclusion, the conditions of CP-divisibility and Ldivisibility for Pauli channels with positive eigenvalues coincide, thus, in this case $\mathrm{C}^{\mathrm{CP}}$ implies $\mathrm{C}^{\mathrm{L}}$.

Concatenating (positive-eigenvalues) Pauli channels with $\mathcal{D}_{x, y, z}$ one can generate the whole set of $\mathrm{C}^{\mathrm{CP}}$ Pauli channels. Using the identity $\mathrm{C}^{\mathrm{CP}}=\mathrm{C}^{\mathrm{Inf}}$ and considering Eq. (11)we can formulate the following theorem:

Theorem 4 (Infinitesimal divisible unital channels). Let $\mathcal{E}_{\text {unital }}^{C P}$ be an arbitrary infinitesimal divisible unital qubit channel. There exists at least one L-divisible Pauli channel $\tilde{\mathcal{E}}$, and two unitary conjugations $\mathcal{U}_{1}$ and $\mathcal{U}_{2}$, such that

$$
\mathcal{E}_{\text {unital }}^{C P}=\mathcal{U}_{1} \tilde{\mathcal{E}} \mathcal{U}_{2}
$$

Notice that if $\mathcal{E}_{\text {unital }}^{C P}$ is invertible, $\tilde{\mathcal{E}}=e^{L}$.

Let us continue with another equivalence relation holding for Pauli channels. Regarding infinitely divisibility channels, we know that, in general, $\mathrm{C}^{\mathrm{L}} \subset \mathrm{C}^{\infty}$, however, for Pauli channels the corresponding subsets coincide.

Theorem 5 (Infinitely divisible Pauli channels). The set of L-divisible Pauli channels is equivalent to the set of infinitely divisible Pauli channels.

Proof. A channel is infinitely divisible if and only if it can be written as $\mathcal{E}_{0} e^{L}$, where $\mathcal{E}_{0}$ is an idempotent channel satisfying $\mathcal{E}_{0} L \mathcal{E}_{0}=\mathcal{E}_{0} L$ and $L$ has

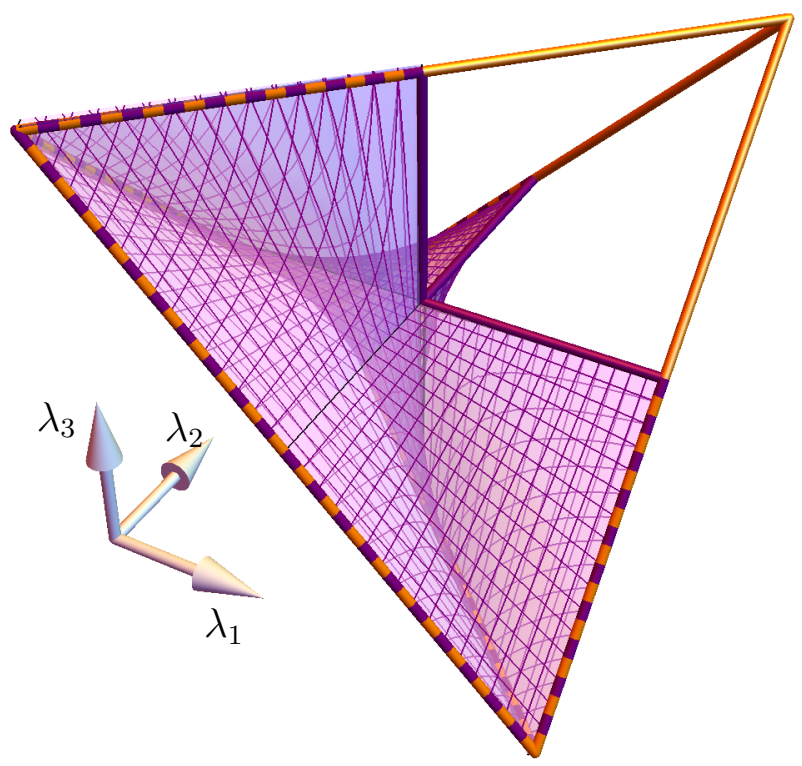

Figure 5: Tetrahedron of Pauli channels with part of the set of CP-divisible, see Eq. (15), but not L-divisible channels $\left(C^{C P} \backslash C^{L}\right)$ shown in purple. The whole set $C^{C P}$ is obtained applying the symmetry transformations of the tetrahedron to the purple volume.

Lindblad form [4]. The only idempotent qubit channels are contractions of the Bloch sphere into single points, diagonalization channels $\mathcal{E}_{\text {diag }}$ transforming Bloch sphere into a line connecting a pair of basis states, and the identity channel. Among the single-point contractions, the only one that is a Pauli channel is the contraction of the Bloch sphere into the complete mixture. In particular, $\mathcal{E}=\mathcal{N} e^{L}=$ $\mathcal{N}$ for all $L$. The channel $\mathcal{N}$ belongs to the closure of $\mathrm{C}^{\mathrm{L}}$, because a sequence of channels $e^{L_{n}}$ with $\hat{L}_{n}=\operatorname{diag}(0,-n,-n,-n)$ converges to $\hat{\mathcal{N}}$ in the limit $n \rightarrow \infty$. For the case of $\mathcal{E}_{0}$ being the identity channel we have $\mathcal{E}=e^{L}$, thus, trivially such infinitely divisible channel $\mathcal{E}$ is in $\mathrm{C}^{\mathrm{L}}$ too. It remains to analyze the case of diagonalization channels. First, let us note that the matrix of $e^{\hat{L}}$ is necessarily of full rank, since $\operatorname{det} \hat{\mathcal{E}} \neq 0$. It follows that the matrix $\hat{\mathcal{E}}=\hat{\mathcal{E}}_{\text {diag }} e^{\hat{L}}$ has rank two as $\hat{\mathcal{E}}_{\text {diag }}$ is a rank two matrix, thus, it takes one of the following forms $\hat{\mathcal{E}}_{x}^{\lambda}=\operatorname{diag}(1, \lambda, 0,0)$, $\hat{\mathcal{E}}_{y}^{\lambda}=\operatorname{diag}(1,0, \lambda, 0), \hat{\mathcal{E}}_{z}^{\lambda}=\operatorname{diag}(1,0,0, \lambda)$. The infinitely divisibility implies $\lambda>0$ in order to keep the roots of $\lambda$ real. In what follows we will show that $\hat{\mathcal{E}}_{z}$ belongs to (the closure of) $\mathrm{C}^{\mathrm{L}}$. Let us define the channels $\hat{\mathcal{E}}_{z}^{\lambda, \epsilon}=\operatorname{diag}(1, \epsilon, \epsilon, \lambda)$ with $\epsilon>0$. The complete positivity and ccp conditions translate into the inequalities $\epsilon \leq \frac{1+\lambda}{2}$ and $\epsilon^{2} \leq \lambda$, respectively; therefore one can always find an $\epsilon>0$ such that $\hat{\mathcal{E}}_{z}^{\lambda, \epsilon}$ is a L-divisible channel. If we choose $\epsilon=\sqrt{\lambda} / n$ with $n \in \mathbb{Z}^{+}$, the channels $\hat{\mathcal{E}}_{z, n}=\operatorname{diag}(1, \sqrt{\lambda} / n, \sqrt{\lambda} / n, \lambda)$ form a sequence of L-divisible channels converging to $\hat{\mathcal{E}}_{z}^{\lambda}$ when $n \rightarrow \infty$. The analogous reasoning implies that $\hat{\mathcal{E}}_{x}^{\lambda}, \hat{\mathcal{E}}_{y}^{\lambda} \in \mathrm{C}^{\mathrm{L}}$ too. Let us note that one parame- 


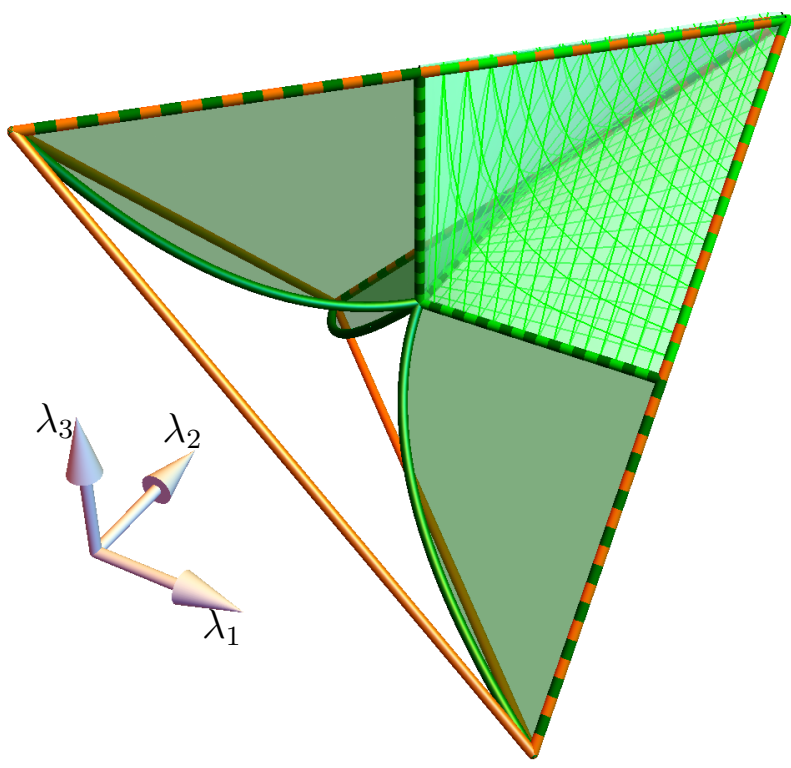

Figure 6: Tetrahedron of Pauli channels with the set of Ldivisible channels (or equivalently infinitely divisible, see Theorem 5) shown in green, see equations (17) and (18). The solid set corresponds to channels with positive eigenvalues, and the $2 \mathrm{D}$ sets correspond to the negative eigenvalue case. The point where the four sets meet corresponds to the total depolarizing channel. Notice that this set does not have the symmetries of the tetrahedron.

ter family $\mathcal{E}_{z}$ are convex combinations of the complete diagonalization channel $\hat{\mathcal{E}}_{z}^{1}=\operatorname{diag}(1,0,0,1)$ and the complete mixture contraction $\hat{\mathcal{N}}$. This completes the proof.

Finally, let us remark that using theorem 23 of Ref. [25] we conclude that the intersection $C^{\mathrm{P}} \cap \overline{\mathrm{C}^{\text {div }}}$ depicted in Fig. 1 is not empty. To show this, notice that applying the mentioned theorem to Pauli channels we get that the faces of the tetrahedron are indivisible in CPTP channels. However, there are channels with positive determinant inside the faces, for example diag $\left(1, \frac{4}{5}, \frac{4}{5}, \frac{3}{5}\right)$. Therefore we conclude that up to unitaries, $C^{\mathrm{P}} \cap \overline{\mathrm{C}^{\text {div }}}$ correspond to the union of the four faces faces of the tetrahedron minus the faces of the octahedron that intersects with the faces of the tetrahedron, see Fig. 3. We have to remove such intersection since it corresponds to channels with negative determinant, i.e. not in $\mathrm{C}^{\mathrm{P}}$.

To get a detailed picture of the position and inclusions of the divisibility sets, we illustrate in Fig. 7 two slices of the tetrahedron where different types of divisibility are visualized. Notice the non-convexity of the considered divisibility sets.

\subsection{Non-unital qubit channels}

Similar to unital channels, using theorem 1 we are able to characterize $\mathrm{C}^{\text {div }}, \mathrm{C}^{\mathrm{P}}$ and $\mathrm{C}^{\mathrm{CP}}$ by studying special orthogonal normal forms of non-unital channels. They are characterized by $\vec{\lambda}$ and $\vec{\tau}$, see Eq. (5).
Thus, we can study if a channel is $\mathrm{C}^{\text {div }}$ by computing the rank of its Choi matrix. For this case algebraic equations are in general fourth order polynomials. In fact, in Ref. [19] a condition in terms of the eigenvalues and $\vec{\tau}$ is given. For special cases, however, we can obtain compact expressions, see Fig. 8. The characterization of $\mathrm{C}^{\mathrm{P}}$ is given by Eq. (8) (note that it only depends on $\vec{\lambda}$ ), and $\mathrm{C}^{\mathrm{CP}}$ is tested, for full Kraus rank non-unital channels, using Eq. (9), see Ref. [24] for the calculation of the $s_{i}$ 's. For the characterization of $\mathrm{C}^{\mathrm{L}}$ we use the results developed at the end of the last section, see Eqs. (18)-(22).

We can plot illustrative pictures even though the whole space of qubit channels has 12 parameters. This can be done using orthogonal normal forms and fixing $\vec{\tau}$, exactly in the same way as the unital case. Recall that unitaries only modify $\mathrm{C}^{\mathrm{L}}$, leaving the shape of other sets unchanged. CPTP channels are represented as a volume inside the tetrahedron presented in Fig. 2, see Fig. 8. In the later figure we show a slice corresponding to $\vec{\tau}=(1 / 2,0,0)^{\mathrm{T}}$. Indeed, it has the same structure of the slices for the unital case, but deformed, see Fig. 7. A difference with respect to the unital case is that L-divisible channels with negative eigenvalues (up to unitaries) are not completely inside CP-divisible channels. A part of them are inside the $\mathrm{C}^{\mathrm{P}}$ channels.

A central feature of Figs. 7 and 8 is that the set $\mathrm{C}^{\text {div }} \backslash \mathrm{C}^{\mathrm{P}}$ is inside the convex slice of the set of entanglement breaking channels (deformed octahedron). Indeed, we can proof the following theorem.

Theorem 6 (Entanglement breaking channels and divisibility). Consider a qubit channel $\mathcal{E}$. If $\operatorname{det} \hat{\mathcal{E}}<0$, then $\mathcal{E}$ is entanglement breaking, i.e. all qubit channels outside $\mathrm{C}^{P}$ are entanglement breaking.

Proof. Consider the Choi-Jamiołkowski state of a channel $\mathcal{E}$ written in the factorized Pauli operator basis $\tau_{\mathcal{E}}=\frac{1}{4} \sum_{j k}^{3} R_{j k} \sigma_{j} \otimes \sigma_{k}$ [24], and let $\hat{\mathcal{E}}$ be its representation in the Pauli operator basis. Then the matrix identity $R=\hat{\mathcal{E}} \Phi_{\mathrm{T}}$ with $\Phi_{\mathrm{T}}=\operatorname{diag}(1,1,-1,1)$ holds. Since $\operatorname{det} \hat{\mathcal{E}}<0$ it follows that $\operatorname{det} R=-\operatorname{det} \hat{\mathcal{E}}>0$. Using the aforementioned Lorentz normal decomposition $R=L_{1}^{\mathrm{T}} \tilde{R} L_{2}$ with $\operatorname{det} L_{1,2}>0$, and $\tilde{R}$ diagonal for $\mathcal{E}$ with full Kraus rank, see Ref. [24]. The transformations $L_{1,2}$ correspond to one-way stochastic local operations and classical communications (1wSLOCC) of $\tau_{\mathcal{E}}$, thus, $\tilde{R}$ corresponds to an unnormalized twoqubit state with det $\tilde{R}>0$. The channel corresponding to $\tilde{R}$ (in the Pauli basis) is $\hat{\mathcal{G}}=\tilde{R} \Phi_{\mathrm{T}} / \tilde{R}_{00}$. Since the latter is diagonal, then $\mathcal{G}$ is a Pauli channel with $\operatorname{det} \hat{\mathcal{G}}<0$. A Pauli channel has a negative determinant if either all $\lambda_{j}$ are negative, or exactly one of them is negative. In Ref. [28] it has been shown that the set of channels with $\lambda_{j}<0 \quad \forall j$ are entanglement breaking channels. Now, using the symmetries of the tetrahedron, one can generate all channels with negative determinant by concatenating this set with the 


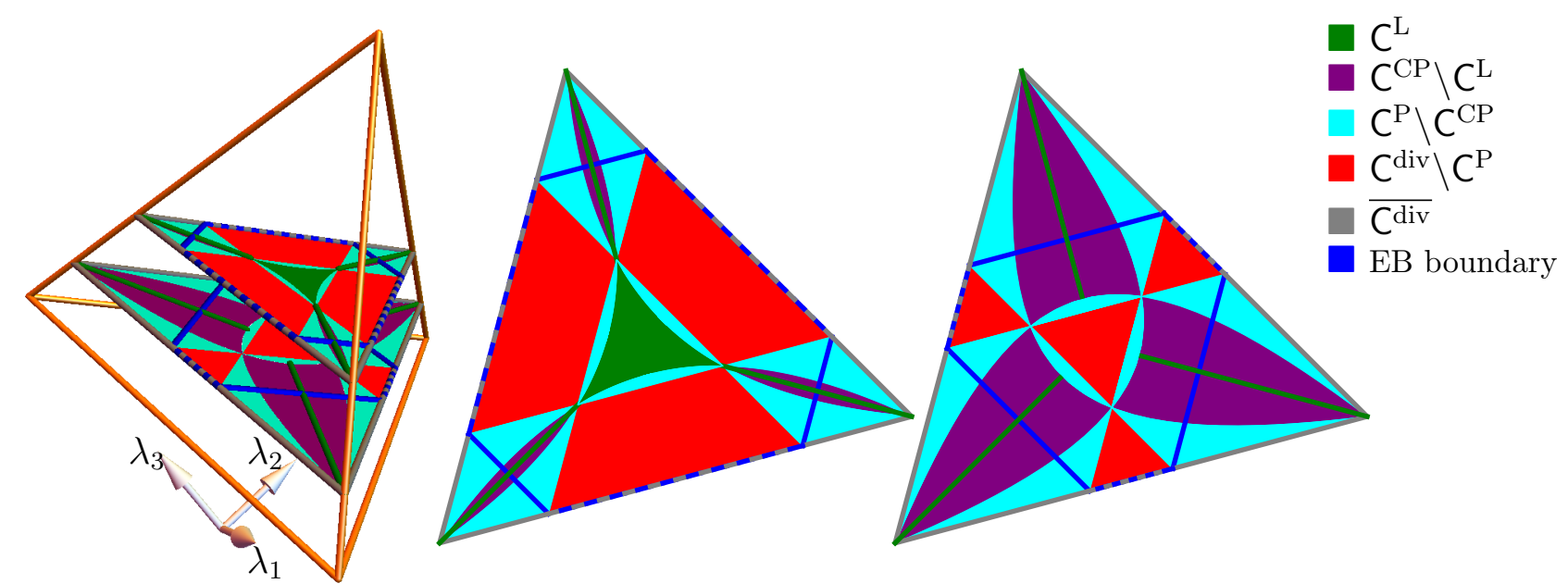

Figure 7: We show two slices of the unitary tetrahedron (figure in the left) determined by $\sum_{i} \lambda_{i}=0.4$ (shown in the center) and $\sum_{i} \lambda_{i}=-0.4$ (shown in the right). The non-convexity of the divisibility sets can be seen, including the set of indivisible channels. The convexity of sets $C$ and entanglement breaking channels can also be noticed in the slices. A central feature is that the set $C^{\text {div }} \backslash C^{P}$ is always inside the octahedron of entanglement breaking channels.

Pauli rotations. Therefore every Pauli channel with negative determinant is entanglement breaking, thus $\tau_{\mathcal{G}}$ is separable. Given that 1 wSLOCC operations can not create entanglement [11], we have that $\tau_{\mathcal{E}}$ is separable too. Therefore $\mathcal{E}$ is entanglement breaking.

The case when $\tilde{R}$ is non-diagonal corresponds to Kraus deficient channels (the matrix rank of Eq. (6) is at most 3$)$. This case can be analyzed as follows. Since the neighborhood of any Kraus deficient channel with negative determinant contains full Kraus rank channels, by continuity of the determinant such channels have negative determinant too. The last ones are entanglement breaking since full Kraus rank channels have diagonal Lorentz normal form. Therefore, by continuity of the concurrence [28], Kraus deficient channels with negative determinant are entanglement breaking.

\section{Divisibility transitions and examples with dynamical process}

The aim of this section is to use illustrative examples of quantum dynamical processes to show transitions between divisibility types of the instantaneous channels. From the slices shown above (see figures 7 and 8) it can be noticed that every transition between the studied divisibility types is permitted. This is due to the existence of common borders between all combinations of divisibility sets; we can think of any continuous line inside the tetrahedron [6] as describing some quantum dynamical map.

We analyze two examples, the first is an implementation of the approximate NOT gate, $\mathcal{A}_{\mathrm{NOT}}$ throughout a specific collision model [21]. The second is the well known setting of a two-level atom interacting

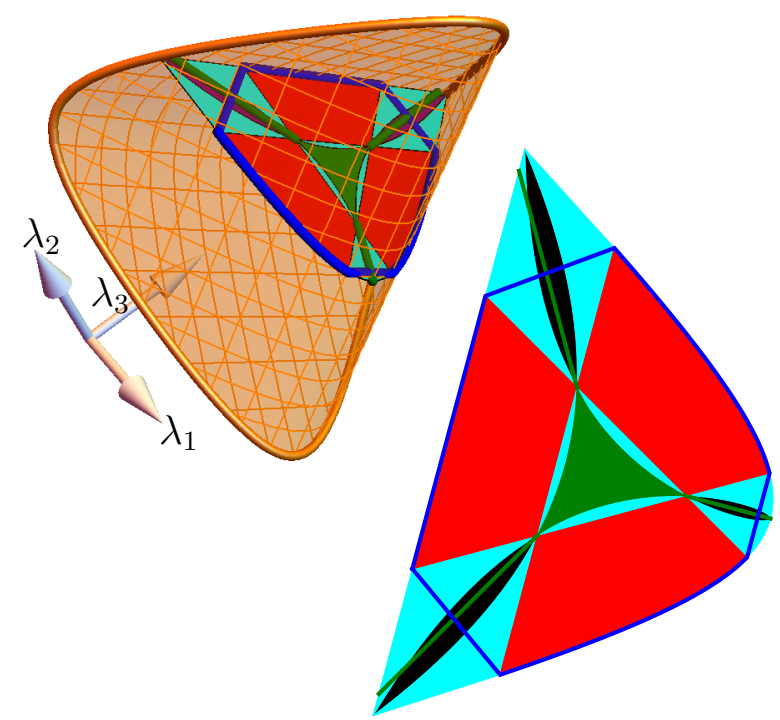

Figure 8: (left) Set of non-unital unital channels up to unitaries, defined by $\vec{\tau}=(1 / 2,0,0)$, see Eq. (5). This set lies inside the tetrahedron. For this particular case the $\mathrm{CP}$ conditions reduce to the two inequalities $2 \pm 2 \lambda_{1} \geq$ $\sqrt{1+4\left(\lambda_{2} \pm \lambda_{3}\right)^{2}}$. A cut corresponding to $\sum_{i} \lambda_{i}=0.3$ is presented inside and in the right, see Fig. 7 for the color coding. The structure of divisibility sets presented here has basically the same structure as for the unital case except for $C^{\mathrm{L}}$. A part of the channels with negative eigenvalues belonging to $C^{\mathrm{L}}$ lies outside $C^{C P} \backslash C^{\mathrm{L}}$, see green lines. As for the unital case a central feature is that the channels in $C^{\text {div }} \backslash C^{P}$ are entanglement breaking channels. Channels in the boundary are not characterized due to the restricted character of Theorem 2. 
with a quantized mode of an optical cavity [9]. We define a simple function that assigns a particular value to a channel $\mathcal{E}_{t}$ according to divisibility hierarchy, i.e.

$$
\delta[\mathcal{E}]=\left\{\begin{array}{cl}
1 & \text { if } \mathcal{E} \in \mathrm{C}^{\mathrm{L}}, \\
2 / 3 & \text { if } \mathcal{E} \in \mathrm{C}^{\mathrm{CP}} \backslash \mathrm{C}^{\mathrm{L}} \\
1 / 3 & \text { if } \mathcal{E} \in \mathrm{C}^{\mathrm{P}} \backslash \mathrm{C}^{\mathrm{CP}} \\
0 & \text { if } \mathcal{E} \in \mathrm{C} \backslash \mathrm{C}^{\mathrm{P}}
\end{array}\right.
$$

A similar function can be defined to study the transition to/from the set of entanglement breaking channels, i.e.

$$
\chi[\mathcal{E}]= \begin{cases}1 & \text { if } \mathcal{E} \text { is entanglement breaking, } \\ 0 & \text { if } \mathcal{E} \text { if not. }\end{cases}
$$

The quantum NOT gate is defined as NOT : $\rho \mapsto$ $\mathbb{1}-\rho$, i.e. it maps pure qubit states to its orthogonal state. Although this map transforms the Bloch sphere into itself it is not a CPTP map, and the closest CPTP map is $\mathcal{A}_{\mathrm{NOT}}: \rho \mapsto(2 \mathbb{1}-\rho) / 3$. This is a rank-three qubit unital channel, thus, it is indivisible [25]. Moreover, $\operatorname{det} \mathcal{A}_{\mathrm{NOT}}=-1 / 27$ implies that this channel is not achievable by a $\mathrm{P}$-divisible dynamical map. It is worth noting that $\mathcal{A}_{\mathrm{NOT}}$ belongs to $\overline{\mathrm{C}^{\mathrm{div}}}$.

A specific collision model was designed in Ref. [21] simulating stroboscopically a quantum dynamical map that implements the quantum NOT gate $\mathcal{A}_{\mathrm{NOT}}$ in finite time. The model reads

$$
\mathcal{E}_{t}(\varrho)=\cos ^{2}(t) \varrho+\sin ^{2}(t) \mathcal{A}_{\mathrm{NOT}}(\varrho)+\frac{1}{2} \sin (2 t) \mathcal{F}(\varrho),
$$

where $\mathcal{F}(\varrho)=i \frac{1}{3} \sum_{j}\left[\sigma_{j}, \varrho\right]$. This quantum dynamical map achieves the desired gate $\mathcal{A}_{\mathrm{NOT}}$ at $t=\pi / 2$.

Let us stress that this dynamical map is unital, i.e. $\mathcal{E}_{t}(\mathbb{1})=\mathbb{1}$ for all $t$, thus, its orthogonal normal form can be illustrated inside the tetrahedron of Pauli channels, see Fig. 9. In Fig. 10 we plot $\delta\left[\mathcal{E}_{t}\right]$, $\chi\left[\mathcal{E}_{t}\right]$ and the value of the $\operatorname{det} \mathcal{E}_{t}$. We see the transitions $\mathrm{C}^{\mathrm{L}} \rightarrow \mathrm{C}^{\mathrm{P}} \backslash \mathrm{C}^{\mathrm{CP}} \rightarrow \mathrm{C}^{\text {div }} \backslash \mathrm{C}^{\mathrm{P}} \rightarrow \overline{\mathrm{C}^{\text {div }}}$ and back. Notice that in both plots the trajectory never goes through the $\mathrm{C}^{\mathrm{CP}} \backslash \mathrm{C}^{\mathrm{L}}$ region. This means that when the parametrized channels up to rotations belong to $\mathrm{C}^{\mathrm{L}}$, so do the original ones. The transition between $\mathrm{P}$-divisible and divisible channels, i.e. $\mathrm{C}^{\mathrm{P}} \backslash \mathrm{C}^{\mathrm{CP}}$ and $\mathrm{C}^{\operatorname{div}} \backslash \mathrm{C}^{\mathrm{P}}$, occurs at the discontinuity in the yellow curve in Fig. 9. Let us note that this discontinuity only occurs in the space of $\vec{\lambda}$; it is a consequence of the orthogonal normal decomposition, see Eq. (5). The complete channel is continuous in the full convex space of qubit CPTP maps. The transition from $\mathrm{C}^{\mathrm{P}} \backslash \mathrm{C}^{\text {div }}$ and back occurs at times $\pi / 3$ and $2 \pi / 3$. It can also be noted that the transition to entanglement breaking channels occurs shortly before the channel enters in the $\mathrm{C}^{\text {div }} \backslash \mathrm{C}^{\mathrm{P}}$ region; likewise, the channel stops being entanglement breaking shortly after it leaves the $\mathrm{C}^{\text {div }} \backslash \mathrm{C}^{\mathrm{P}}$ region.

Consider now the dynamical map induced by a twolevel atom interacting with a mode of a boson field. This model serves as a workhorse to explore a great

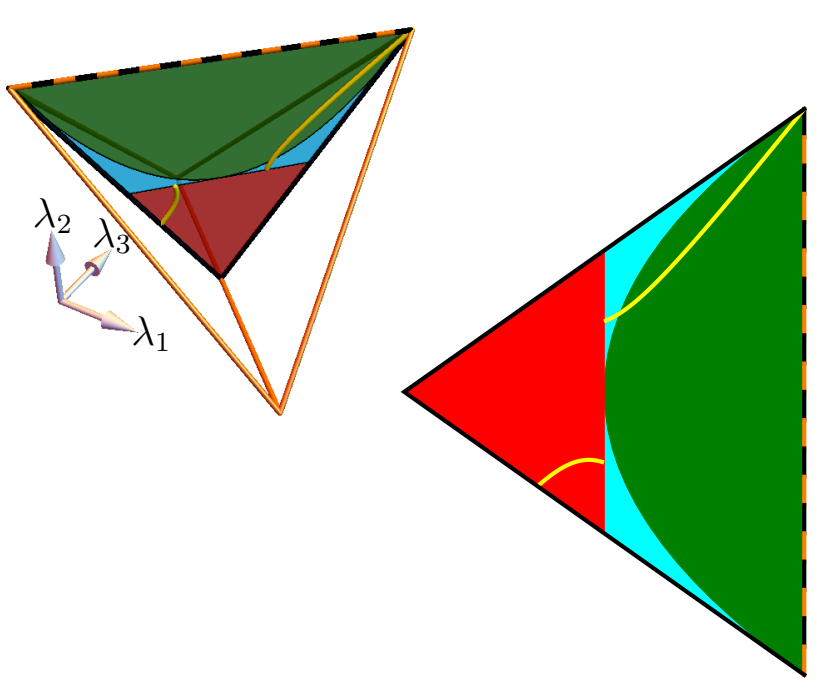

Figure 9: (top left) Tetrahedron of Pauli channels with the trajectory, up to rotations, of the quantum dynamical map Eq. (25) leading to the $\mathcal{A}_{\text {NOT }}$ gate, as a yellow curve. (right) Cut along the plane that contains the trajectory; there one can see the different regions where the channel passes. For this case, the characterization of the $C^{\mathrm{L}}$ of the channels induced gives the same conclusions as for the corresponding Pauli channel, see Eq. (5). The discontinuity in the trajectory is due to the reduced representation of the dynamical map, see Eq. (25); the trajectory is continuous in the space of channels. See Fig. 7 for the color coding.

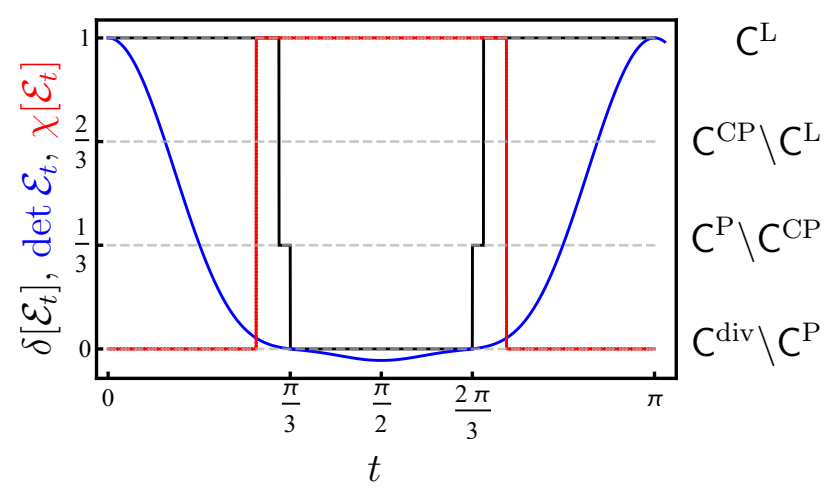

Figure 10: Evolution of divisibility, determinant, and entanglement breaking properties of the map induced by Eq. (25), see Eq. (23) and Eq. (24). Notice that the channel $\mathcal{A}_{\text {NOT }}$, implemented at $t=\pi / 2$, has minimum determinant. The horizontal gray dashed lines show the image of the function $\delta$, with the divisibility types in the right side. It can be seen that the dynamical map explores the divisibility sets as $\mathrm{C}^{\mathrm{L}} \rightarrow \mathrm{C}^{\mathrm{P}} \backslash \mathrm{C}^{\mathrm{CP}} \rightarrow \mathrm{C}^{\text {div }} \backslash \mathrm{C}^{\mathrm{P}} \rightarrow \overline{\mathrm{C}^{\text {div }}}$ and back. The channels are entanglement breaking in the expected region. 


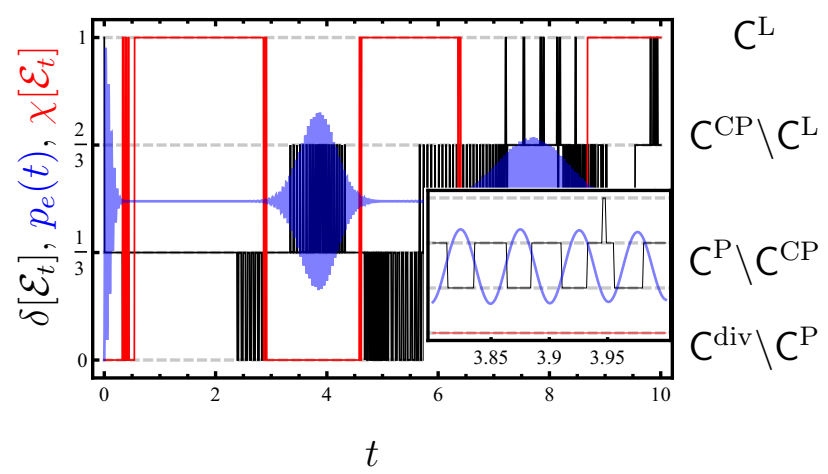

Figure 11: Black and red curves show functions $\delta$ and $\chi$ of the channels induced by the Jaynes-Cummings model over a twolevel system, see Eq. (26) with the environment initialized in a coherent state $|\alpha\rangle$. The blue curve shows the probability of finding the two-level atom in its excited state, $p_{e}(t)$. The figure shows that the fast oscillations in $\delta$ occur roughly at the same frequency as the ones of $p_{e}(t)$, see the inset. Notice that there are fast transitions between $C^{\mathrm{P}} \backslash \mathrm{C}^{\mathrm{CP}}$ and $\mathrm{C}^{\mathrm{CP}} \backslash \mathrm{C}^{\mathrm{L}}$ occurring in the region of revivals, with a few transitions between $C^{C P} \backslash C^{P}$ and $C^{L}$ in the second revival. The function $\chi$ shows that during revivals channels are not entanglement breaking, but we find that channels belonging to $C^{\text {div }} \backslash C^{P}$ are always entanglement breaking, in agreement with theorem 6 . The particular chosen set of parameters are $\alpha=6, g=10$, $\omega_{a}=5$, and $\omega_{f}=20$.

variety of phenomena in quantum optics [8]. Using the well known rotating wave approximation one arrives to the Jaynes-Cummings model [12], whose Hamiltonian is

$$
H=\frac{\omega_{a}}{2} \sigma_{z}+\omega_{f}\left(a^{\dagger} a+\frac{1}{2}\right)+g\left(\sigma_{-} a^{\dagger}+\sigma_{+} a\right) .
$$

By initializing the environment in a coherent state $|\alpha\rangle$, one gets the familiar collapse and revival setting. Considering a particular set of parameters shown in Fig. 11, we constructed the channels parametrized by time numerically, and studied their divisibility and entanglement breaking properties. In the same figure we plot functions $\delta\left[\mathcal{E}_{t}\right]$ and $\chi\left[\mathcal{E}_{t}\right]$, together with the probability of finding the atom in its excited state $p_{e}(t)$, to study and compare the divisibility properties with the features of the collapses and revivals. The probability $p_{e}(t)$ is calculated choosing the ground state of the free Hamiltonian $\omega_{a} / 2 \sigma_{z}$ of the qubit, and it is given by [13]:

$$
p_{e}(t)=\frac{\left\langle\sigma_{z}(t)\right\rangle+1}{2},
$$

where

$\left\langle\sigma_{z}(t)\right\rangle=-\sum_{n=0}^{\infty} P_{n}\left(\frac{\Delta^{2}}{4 \Omega_{n}^{2}}+\left(1-\frac{\Delta^{2}}{4 \Omega_{n}^{2}}\right) \cos \left(2 \Omega_{n} t\right)\right)$,

with $P_{n}=e^{-|\alpha|^{2}}|\alpha|^{2 n} / n !, \Omega_{n}=\sqrt{\Delta^{2} / 4+g^{2} n}$ and $\Delta=\omega_{f}-\omega_{a}$ the detuning.

The divisibility indicator function $\delta$ exhibits an oscillating behavior, roughly at the same frequency of $p_{e}(t)$, see inset in Fig. 11. The figure shows fast periodic transitions between $C^{\mathrm{P}} \backslash \mathrm{C}^{\mathrm{CP}}$ and $\mathrm{C}^{\mathrm{CP}} \backslash \mathrm{C}^{\mathrm{L}}$ occurring in the region of revivals. There are also few transitions among $\mathrm{CPP}^{\mathrm{CP}} \backslash \mathrm{C}^{\mathrm{P}}$ and $\mathrm{C}^{\mathrm{L}}$ in the second revival. Respect to the entanglement breaking and the function $\chi$, there are no fast transitions in the former, and during revivals, channels are not entanglement breaking. We also observe that channels belonging to $C^{\text {div }} \backslash C^{P}$ are entanglement breaking, supporting theorem 6 for the non-unital case.

\section{Conclusions}

We studied the relations between different types of divisibility of time-discrete and time-continuous quantum processes, i.e. channels and dynamical maps, respectively. In particular, we investigated classes of channels by means of their achievability by dynamical maps of different divisibility types, and also the divisibility of channels occurring during the time evolutions. Apart from investigating the relations between these concepts in general, we provided a detailed analysis for the case of qubit channels.

We implemented the known conditions to decide $\mathrm{C}^{\mathrm{L}}$ for the general diagonalizable case, and a discussion of the parametric space of Lindblad generators was given (clarifying one of the results of the paper [26]). For unital qubit channels it was shown that every infinitesimal divisible map can be written as a concatenation of one $\mathrm{C}^{\mathrm{L}}$ channel and two unitary conjugations. For the particular case of Pauli channels case, we have shown that the sets of infinitely divisible and L-divisible channels coincide. We made an interesting observation, connecting the concept of divisibility with the quantum information paradigm of entanglement breaking channels. We found that divisible but not infinitesimal divisible qubit channels, in PTP maps, are necessarily entanglement breaking. We also noted that the intersection of indivisible and P-divisible channels is not empty. This allows us to implement indivisible channels with infinitesimal PTP maps. Finally, we questioned the existence of dynamical transitions between different classes of divisibility channels. We argued that all the transitions are, in principle, possible, and exploited two simple models of dynamical maps to demonstrate these transitions. They clearly illustrate how the channels evolutions change from being implementable by markovian dynamical maps to non-markovian, and vice versa.

There are several directions how to proceed further in investigation of divisibility of channels and dynamical maps. Apart from extension of this analysis to larger-dimensional systems, a plethora of interesting questions are related to design of efficient verification procedures of the divisibility classes for channels and dynamical maps. In this paper we question the divisibility features of snapshots of the evolution, however, it might be of interest to understand when the time 
intervals of dynamical maps implemented by nonmarkovian evolutions, can be simulated by markovian dynamical maps. Also the area of channel divisibility contains several open structural questions, e.g. the existence of at most $n$-divisible channels.

\section{Acknowledgements}

We acknowledge Thomas Gorin and Tomáš Rybár for useful discussions, as well PAEP and RedTC for financial support. Support by projects CONACyT 285754, UNAM-PAPIIT IG100518, IN-107414, APVV-140878 (QETWORK) is acknowledged. CP acknowledges support by PASPA program from DGAPAUNAM. MZ acknowledges the support of VEGA 2/0173/17 (MAXAP), GAČR project no. GA1622211S and MUNI/G/1211/2017 (GRUPIK).

\section{A On Lorentz normal forms of Choi- Jamiolkowski state}

In this appendix we compute the Lorentz normal decomposition of a channel for which one gets $b \neq 0$, supporting our observation that Lorentz normal decomposition does not take Choi-Jamiołkowski states to something proportional to a Choi-Jamiołkowski state. Consider the following Kraus rank three channel and its $R_{\mathcal{E}}$ matrix, both written in the Pauli basis:

$$
\hat{\mathcal{E}}=\left(\begin{array}{cccc}
1 & 0 & 0 & 0 \\
0 & -\frac{1}{3} & 0 & 0 \\
0 & 0 & -\frac{1}{3} & 0 \\
\frac{2}{3} & 0 & 0 & \frac{1}{3}
\end{array}\right)
$$

and

$$
R_{\mathcal{E}}=\left(\begin{array}{cccc}
1 & 0 & 0 & 0 \\
0 & -\frac{1}{3} & 0 & 0 \\
0 & 0 & \frac{1}{3} & 0 \\
\frac{2}{3} & 0 & 0 & \frac{1}{3}
\end{array}\right)
$$

Using the algorithm introduced in Ref. [24] to calculate $R_{\mathcal{E}}$ 's Lorentz decomposition into orthochronous proper Lorentz transformations we obtain

$$
L_{1}=\frac{1}{\gamma_{1}}\left(\begin{array}{cccc}
4 & 0 & 0 & 1 \\
0 & -\gamma_{1} & 0 & 0 \\
0 & 0 & -\gamma_{1} & 0 \\
1 & 0 & 0 & 4
\end{array}\right), \quad(30)
$$

and

$$
\Sigma_{\mathcal{E}}=\frac{1}{\gamma_{3}}\left(\begin{array}{cccc}
\sqrt{11+\frac{109}{\sqrt{97}}} & 0 & 0 & -\frac{\sqrt{97}+1}{\sqrt{89 \sqrt{97}+873}} \\
0 & -\frac{\gamma_{3}}{3} & 0 & 0 \\
0 & 0 & \frac{\gamma_{3}}{3} & 0 \\
\sqrt{1+\frac{49}{\sqrt{97}}} & 0 & 0 & \sqrt{-1+\frac{49}{\sqrt{97}}}
\end{array}\right)
$$

with $\gamma_{1}=\sqrt{15}, \gamma_{2}=3 \sqrt{178 \sqrt{97}+1746}$, and $\gamma_{3}=$ $\sqrt{30}$. Although the central matrix $\Sigma_{\mathcal{E}}$ is not exactly of the form Eq. (6), it is equivalent. To see this notice that the derivation of the theorem 2 in [24] considers only decompositions into proper orthochronous Lorentz transformations. But to obtain the desired form, the authors change signs until they get Eq. (6); this cannot be done without changing Lorentz transformations. If we relax the condition over $L_{1,2}$ of being proper and orthochronous, we can bring $\Sigma_{\mathcal{E}}$ to the desired form by conjugating $\Sigma_{\mathcal{E}}$ with $G=$ $\operatorname{diag}(1,1,1,-1)$ :

$$
\begin{aligned}
& G^{-1} \Sigma_{\mathcal{E}} G= \\
& \quad \frac{1}{\gamma_{3}}\left(\begin{array}{cccc}
\sqrt{11+\frac{109}{\sqrt{97}}} & 0 & 0 & \frac{\sqrt{97}+1}{\sqrt{89 \sqrt{97}+873}} \\
0 & -\frac{\gamma_{3}}{3} & 0 & 0 \\
0 & 0 & \frac{\gamma_{3}}{3} & 0 \\
-\sqrt{1+\frac{49}{\sqrt{97}}} & 0 & 0 & \sqrt{-1+\frac{49}{\sqrt{97}}}
\end{array}\right) .
\end{aligned}
$$

In both cases (taking $\Sigma_{\mathcal{E}}$ or $G^{-1} \Sigma_{\mathcal{E}} G$ as the normal form of $R_{\mathcal{E}}$ ), the corresponding channel is not proportional to a trace-preserving one since $b \neq 0$, see Eq. (6). This completes the counterexample.

\section{References}

[1] Ángel Rivas, Susana F Huelga, and Martin B Plenio. Quantum non-markovianity: characterization, quantification and detection. Rep. Prog. Phys., 77(9):094001, 2014. DOI: 10.1088/00344885/77/9/094001.

[2] I. Bengtsson and K. Życzkowski. Geometry of Quantum States: An Introduction to Quantum Entanglement. Cambridge University Press, 2017. ISBN 9781107026254. URL https://books.google.com.mx/books?id= sYswDwAAQBAJ.

[3] W. J Culver. On the Existence and Uniqueness of the Real Logarithm of a Matrix. Proceedings of the American Mathematical Society, 17 (5):1146-1151, 1966. DOI: 10.1090/S0002-99391966-0202740-6.

[4] L. V. Denisov. Infinitely Divisible Markov Mappings in Quantum Probability Theory. Theory Prob. Appl., 33(2):392-395, 1989. DOI: $10.1137 / 1133064$.

[5] D. E. Evans and J. T. Lewis. Dilations of Irreversible Evolutions in Algebraic Quantum Theory, volume 24 of Communications of the 
Dublin Institute for Advanced Studies: Theoretical physics. Dublin Institute for Advanced Studies, 1977. URL http://orca.cf.ac.uk/34031/.

[6] S. N. Filippov, J. Piilo, S. Maniscalco, and M. Ziman. Divisibility of quantum dynamical maps and collision models. Phys. Rev. A, 96(3):032111, 2017. DOI: $10.1103 /$ PhysRevA.96.032111.

[7] V. Gorini, A. Kossakowski, and E. C. G. Sudarshan. Completely positive dynamical semigroups of N-level systems. J. Math. Phys., 17(5):821, 1976. DOI: $10.1063 / 1.522979$.

[8] A. D. Greentree, J. Koch, and J. Larson. Fifty years of Jaynes-Cummings physics. J. Phy. $B$, 46(22):220201, 2013 . DOI: 10.1088/09534075/46/22/220201.

[9] S. Haroche and J.-M. Raimond. Exploring the Quantum: Atoms, Cavities, and Photons. Oxford University Press, USA, 2006. URL http: //www.worldcat.org/isbn/0198509146.

[10] T. Heinosaari and M. Ziman. The Mathematical Language of Quantum Theory: From Uncertainty to Entanglement. Cambridge University Press, 2012. DOI: 10.1017/CBO9781139031103.

[11] R. Horodecki, P. Horodecki, M. Horodecki, and K. Horodecki. Quantum entanglement. Rev. Mod. Phys., 81(2):865-942, 2009. DOI: 10.1103/RevModPhys.81.865.

[12] E. T. Jaynes and F. W. Cummings. Comparison of quantum and semiclassical radiation theories with application to the beam maser. Proc. IEEE, 51:89, 1963. DOI: 10.1109/PROC.1963.1664.

[13] A. B. Klimov and S. M. Chumakov. A GroupTheoretical Approach to Quantum Optics: Models of Atom-Field Interactions. Wiley-VCH, 2009. DOI: $10.1002 / 9783527624003$.

[14] A. Kossakowski. On quantum statistical mechanics of non-hamiltonian systems. Rep. Math. Phys., 3(4):247 - 274, 1972. DOI: 10.1016/00344877(72)90010-9.

[15] J. M. Leinaas, J. Myrheim, and E. Ovrum. Geometrical aspects of entanglement. Phys. Rev. A, 74:012313, Jul 2006. DOI: 10.1103/PhysRevA.74.012313.

[16] G. Lindblad. On the generators of quantum dynamical semigroups. Comm. Math. Phys., 48(2): 119-130, 1976. DOI: 10.1007/BF01608499.

[17] M. Musz, M. Kuś, and K. Życzkowski. Unitary quantum gates, perfect entanglers, and unistochastic maps. Phys. Rev. A, 87:022111, Feb 2013. DOI: 10.1103/PhysRevA.87.022111.

[18] C. Pineda, T. Gorin, D. Davalos, D. A. Wisniacki, and I. García-Mata. Measuring and using non-Markovianity. Phys. Rev. A, 93:022117, 2016. DOI: 10.1103/PhysRevA.93.022117.

[19] Ł. Rudnicki, Z. Puchała, and K. Zyczkowski. Gauge invariant information concerning quantum channels. Quantum, 2:60, April 2018. ISSN 2521-327X. DOI: 10.22331/q-2018-04-11-60.
[20] M. B. Ruskai, S. Szarek, and E. Werner. An analysis of completely-positive trace-preserving maps on $\mathrm{M}_{2}$. Lin. Alg. Appl., 347(1):159 - 187, 2002. DOI: 10.1016/S0024-3795(01)00547-X.

[21] T. Rybár, S. N. Filippov, M. Ziman, and V. Bužek. Simulation of indivisible qubit channels in collision models. J. Phys. B, 45(15):154006, 2012. DOI: $10.1088 / 0953-$ $4075 / 45 / 15 / 154006$.

[22] B. Vacchini, A. Smirne, E.-M. Laine, J. Piilo, and H.-P. Breuer. Markovianity and nonmarkovianity in quantum and classical systems. New J. Phys., 13(9):093004, 2011. DOI: 10.1088/1367-2630/13/9/093004.

[23] F. Verstraete and H. Verschelde. On quantum channels. Unpublished, 2002. URL http: //arxiv.org/abs/quant-ph/0202124.

[24] F. Verstraete, J. Dehaene, and B. DeMoor. Local filtering operations on two qubits. Phys. Rev. A, 64(1):010101, 2001. DOI: 10.1103/PhysRevA.64.010101.

[25] M. M. Wolf and J. I. Cirac. Dividing quantum channels. Comm. Math. Phys., 279(1):147-168, 2008. DOI: $10.1007 / \mathrm{s} 00220-008-0411-y$.

[26] M. M. Wolf, J. Eisert, T. S. Cubitt, and J. I. Cirac. Assessing non-Markovian quantum dynamics. Phys. Rev. Lett., 101(15):150402, 2008. DOI: 10.1103/PhysRevLett.101.150402.

[27] M. Ziman and V. Bužek. All (qubit) decoherences: Complete characterization and physical implementation. Phys. Rev. A, 72:022110, Aug 2005. DOI: 10.1103/PhysRevA.72.022110.

[28] M. Ziman and V. Bužek. Concurrence versus purity: Influence of local channels on Bell states of two qubits. Phys. Rev. A, 72(5):052325, 2005. DOI: $10.1103 /$ PhysRevA.72.052325. 